\title{
High intraocular pressure in eyes with late in-the-bag
}

\section{intraocular lens dislocation}

Olav Kristianslund, MD, PhD,1 Marius Dalby, MD,1,2 and Liv Drolsum, MD, $\mathrm{PhD}_{1,2}$

1 Department of Ophthalmology, Oslo University Hospital, Oslo, Norway

2 Institute of clinical medicine, University of Oslo, Oslo, Norway

Running title: IOL dislocation and high IOP

Corresponding author: Olav Kristianslund, Department of Ophthalmology, Oslo University Hospital. Mailbox 4956 Nydalen, 0424 Oslo, Norway.

Phone number: +47 230151 68. Email: olakri@ ous-hf.no

Funding: This work has been partly funded by the Norwegian ExtraFoundation for Health and Rehabilitation, Oslo, Norway (grant number: 2015/FO8068) and the South-Eastern Norway Regional Health Authority, Norway (grant number: 2017020). The funding organizations had no role in the design or conduct of this research and have no related commercial interests. The work was carried out at the Department of Ophthalmology, Oslo University Hospital

Conflict of interest: No author has a financial or proprietary interest in any product, material or method mentioned. 
The frequency of late in-the-bag intraocular lens (IOL) dislocation has increased in the last decades. Important predisposing factors are pseudoexfoliation syndrome (PEX), myopia, previous vitreoretinal surgery, and trauma,1-3 and there is also an association with glaucoma.14 Furthermore, some studies have reported high intraocular pressure (IOP) in IOL dislocation eyes despite no known glaucoma.1-5 Accordingly, it has been speculated if the dislocation can cause an IOP increase.

In a recent study addressing the association between high IOP and spontaneous in-the-bag IOL dislocation, Bulnes et al found a significant trend towards higher IOP when a lower grade of dislocation was diagnosed.6 They suggested that movement of the loosened IOL in mild dislocation may cause iris chafing. The aim of the present analysis was to evaluate this possible association between IOP at diagnosis and grade of IOL dislocation in a quite large clinical trial.

\section{METHODS}

Patients with late in-the-bag IOL dislocation were randomly assigned to either IOL repositioning by scleral suturing $(n=54)$ or IOL exchange with an iris-claw IOL $(n=50)$. The present analysis included all study eyes regardless of operation group. Most patients were referred from private ophthalmologists or other ophthalmology departments, thus, the IOP at diagnosis was gathered from referral notes. All patients had a standardized preoperative study examination.1,7 A flow chart, inclusion criteria, surgical techniques, baseline characteristics, and 6-month outcomes have been previously published.1,7 Ethics 
Committee approval was obtained and the study was registered at ClinicalTrials.gov (identifier, NCT01784926) and conducted in adherence with the Declaration of Helsinki.

The degree of dislocation was evaluated both with slit-lamp examination and images. The classification was: grade 1: small decentration with the IOL optic covering the visual axis, often with pseudophakodonesis; grade 2: the equator of the optic approximately in the visual axis; grade 3: the IOL more decentered than grade 2 but at least one haptic visible in the pupillary area. Grade 4, totally dislocated IOLs, were excluded.1 In cases of uncertainty, two of the authors (O.K. and L.D.) reached a consensus.

\section{RESULTS}

Mean IOP at diagnosis was $20.6 \pm 9.4 \mathrm{mmHg}$ (range, $5-50 \mathrm{mmHg}$ ) for all study eyes $(\mathrm{n}=$ 104), and there were no statistically significant differences between the grades of dislocation $(P=.24)$ as shown in Table 1 and Figure 1. A linear regression analysis with IOP at diagnosis as the dependent variable revealed no statistical significance for any of the included explanatory variables: age $(P=.22)$, gender $(P=.89)$, grade of dislocation $(P=.24)$, interval from cataract surgery to dislocation $(P=.79)$, PEX $(P=.20)$, myopia $(P=.36)$, previous vitreoretinal surgery $(P=.23)$, or recent trauma $(P=.38)$.

\section{DISCUSSION}

High IOP at diagnosis was common in this clinical trial of late in-the-bag IOL dislocation.7 There was a slight tendency towards higher IOP for dislocation grade 1, however, not 
statistically significant. A recent study presented significant results for this trend, albeit limited by few mild dislocations $(n=8)$ with large spread in IOP.6 Although our study had a higher number of mild dislocations, the classifications used were not exactly the same as in the other study, which included only pseudophakodonesis in grade 1.6 We hypothesize that the shown tendency is biased by timing of referral, as several patients with pseudophakodonesis or mild dislocation may go undetected or be followed regularly until a high IOP or progression in dislocation evolve and the decision for surgery is made.

Bulnes et al speculate whether iris chafing and uveitis-glaucoma-hyphema (UGH) syndrome may be the reason for high IOP in these patients.6 Only one UGH syndrome was diagnosed in our study, 7 although this does not preclude that a milder form of iris chafing contributes to the IOP increase. Jakobsson et al hypothesized whether anatomical changes with prolapse of the anterior vitreous surface are involved 4 and it has otherwise been suggested that the dislocation and the high IOP are concurrent results of advanced stage PEX.5,7

In the present study, males typically presented at more advanced stages of dislocation than females, indicating gender differences for consulting an ophthalmologist. This has correspondingly been suggested to partly explain gender differences in cataract surgery. 8

In summary, this analysis revealed no significant association between grade of IOL dislocation and IOP. Analyses from larger patient materials are required to reach certain conclusions regarding mechanisms for increased IOP in these eyes. 


\section{REFERENCES}

1. Kristianslund O, Råen M, Østern AE, Drolsum L. Late In-the-Bag Intraocular Lens Dislocation: A Randomized Clinical Trial Comparing Lens Repositioning and Lens Exchange. Ophthalmology 2017; 124:151-159

2. Lorente R, de Rojas V, Vazquez de Parga P, Moreno C, Landaluce ML, Dominguez R, Lorente B. Management of late spontaneous in-the-bag intraocular lens dislocation: Retrospective analysis of 45 cases. J Cataract Refract Surg 2010; $36: 1270-1282$

3. Shingleton BJ, Yang Y, O'Donoghue MW. Management and outcomes of intraocular lens dislocation in patients with pseudoexfoliation. J Cataract Refract Surg 2013; 39:984-993

4. Jakobsson G, Zetterberg M, Sundelin K, Stenevi U. Surgical repositioning of intraocular lenses after late dislocation: complications, effect on intraocular pressure, and visual outcomes. J Cataract Refract Surg 2013; 39:1879-1885

5. Leung VC, Singh H, Ahmed, II. Inter-eye differences in patients with pseudoexfoliation syndrome presenting with intraocular lens dislocation. Ophthalmology 2015; 122:480-485

6. Bulnes BL, de Rojas Silva MV, Moore RL. Intraocular pressure changes before and after surgery for spontaneous in-the-bag intraocular lens dislocation. J Cataract Refract Surg 2018

7. Kristianslund O, Råen M, Østern AE, Drolsum L. Glaucoma and Intraocular Pressure in Patients Operated for Late In-the-bag Intraocular Lens Dislocation: A Randomized Clinical Trial. Am J Ophthalmol 2017; 176:219-227

8. Mönestam E, Wachtmeister L. Cataract surgery from a gender perspective--a population based study in Sweden. Acta Ophthalmol Scand 1998; 76:711-716 


\begin{tabular}{|c|c|c|c|c|c|}
\hline & All $(n=104)$ & Grade $1(n=36)$ & Grade 2 (n=27) & Grade $3(n=41)$ & $P^{*}$ \\
\hline Age (years) & $81.7 \pm 8.0$ & $80.4 \pm 8.9$ & $83.3 \pm 7.4$ & $81.7 \pm 7.5$ & .38 \\
\hline Gender (male / female) & $41(39) / 63$ (61) & $12(33) / 24(67)$ & $6(22) / 21(78)$ & $23(56) / 18(44)$ & .01 \\
\hline Pseudoexfoliation syndrome & $86(83)$ & $27(75)$ & $24(89)$ & $35(85)$ & .33 \\
\hline Trauma & $10(10)$ & $2(6)$ & $2(7)$ & $6(15)$ & .34 \\
\hline Preexisting glaucoma & $39(38)$ & $11(31)$ & $16(59)$ & $12(29)$ & .03 \\
\hline IOP at diagnosis $(\mathrm{mmHg})$ & $\begin{array}{l}20.6 \pm 9.4 \\
(5-50)\end{array}$ & $\begin{array}{l}22.7 \pm 9.7 \\
(10-50)\end{array}$ & $\begin{array}{l}18.9 \pm 9.1 \\
(5-43)\end{array}$ & $\begin{array}{l}19.9 \pm 9.1 \\
(7-45)\end{array}$ & .24 \\
\hline $\mathrm{IOP} \geq 22 \mathrm{mmHg}$ at diagnosis & $37(36)$ & $16(44)$ & $7(26)$ & $14(34)$ & .33 \\
\hline
\end{tabular}




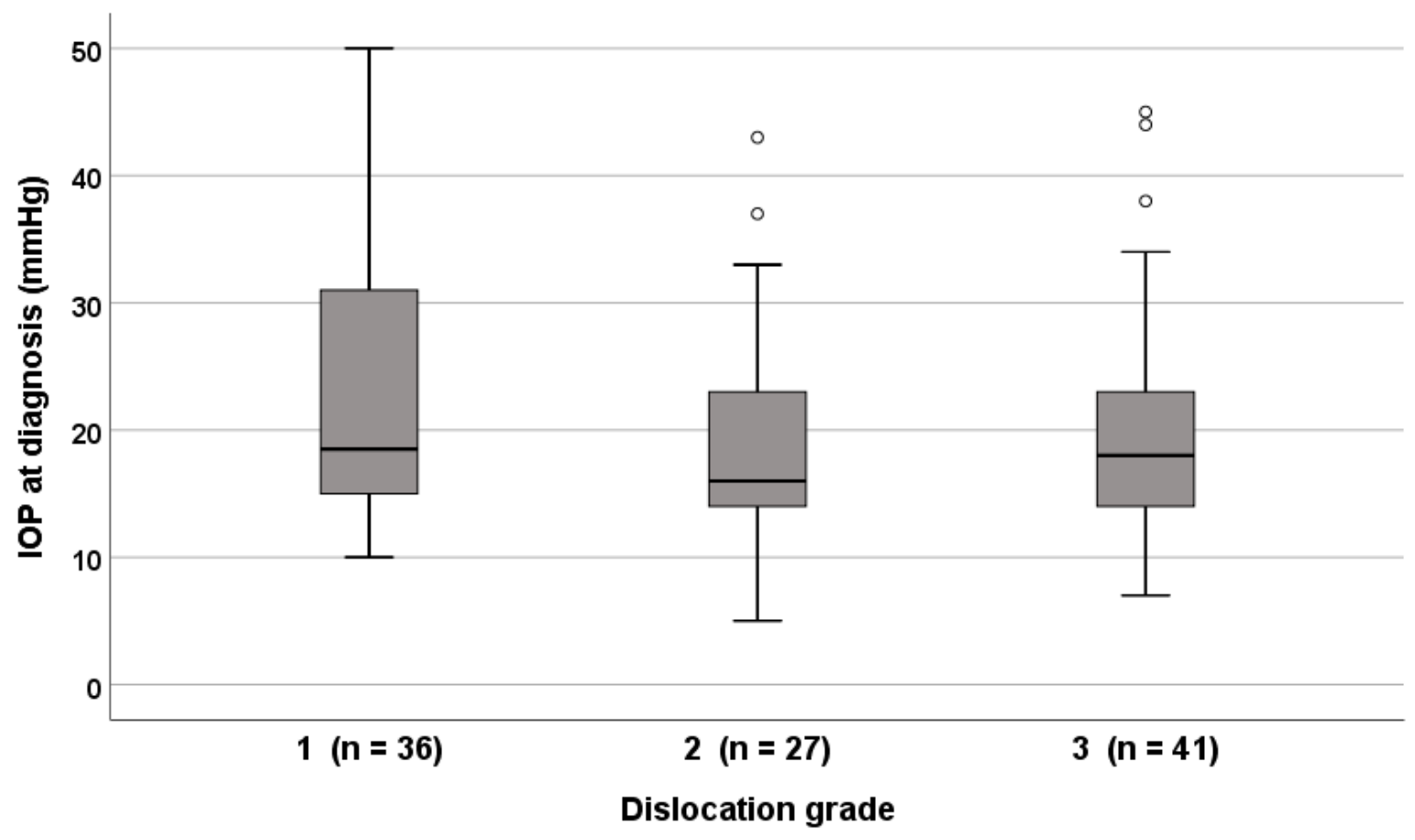

Figure 1. Box plots of intraocular pressure (IOP) at diagnosis of late in-the-bag intraocular lens dislocation, by grade of dislocation. 